DOI:

10.1038/nrn2143

\title{
(Light) remote control of the brain
}

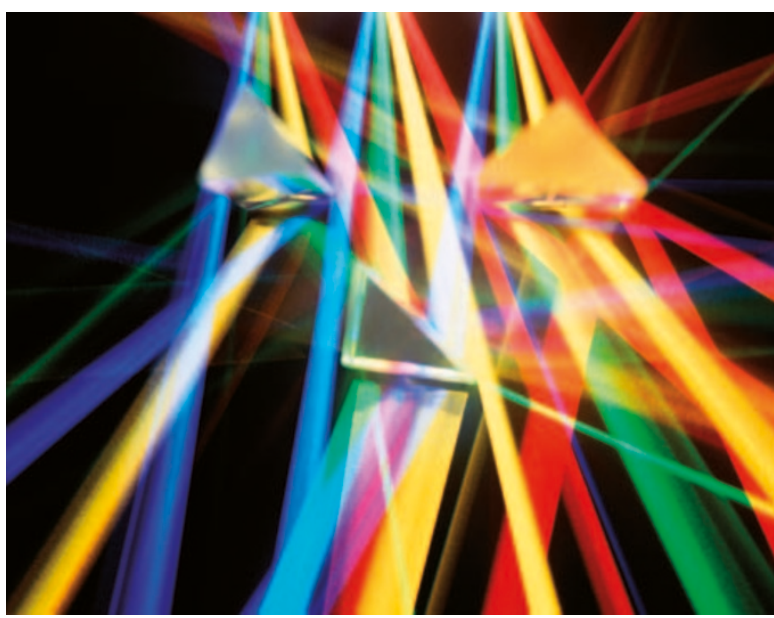

Non-invasive methods to study neuronal circuits have so far seemed out of reach. With their recent publication in Nature, Deisseroth and colleagues have now made the first step towards such technology by employing two light-activated proteins which allowed them to remotely control the activity of rat hippocampal neurons and the behaviour of the nematode Caenorhabditis elegans.

Proteins which alter membrane potential can regulate neuronal activity. As channelrhodopsin 2 (ChR2), an algal cation channel which opens upon exposure to blue light, can be used as an optically excitable and depolarizing channel in neuronal cells, the authors looked for a complementary inhibiting channel that was able to hyperpolarize neuronal membranes with a different spectrum of light. This search lead them to halorhodopsin of the archaea Natronomonas pharaonis (NpHR), a chloride pump activated by yellow light. It is worth noting that ChR2 and NpHR are both naturally occurring proteins found in algae and archaea, but are not expressed in higher-order species.

$\mathrm{NpHR}$ expression in cultured rat hippocampal neurons did not alter their survival or electrical properties. Stimulating NpHR and ChR2 co-expressing neurons with blue light resulted in the firing of action potentials, which were inhibited by yellow light activating NpHR. The degree of inhibition was dependent on the intensity and duration of yellow light exposure. The temporal resolution of this inhibition was so high that brief pulses of yellow light were able to 'knockout' single action potentials, demonstrating for the first time that neuronal activity can be controlled remotely, bidirectionally and with high temporal precision using light. Next, the authors used lentivirus to transfect the brain of mouse pups and the hippocampi of adult mice with
NpHR and ChR2, and showed that neuronal activity in acute cortical and hippocampal slices could also be manipulated by yellow and blue light. In addition, after loading cortical slices with the $\mathrm{Ca}^{2+}$-indicator Fura-2, the authors had an all-optical system at hand in which they were able to fire or block action potentials by pulses of blue or yellow light and monitor intracellular $\mathrm{Ca}^{2+}$ concentration simultaneously.

Furthermore, when both proteins were expressed in either the bodywall muscles or the cholinergic neurons controlling these muscles in C. elegans, Deisseroth and colleagues were able to optically control nematode swimming behaviour, leaving no doubt that ChR2 and NpHR constitute a bidirectional module for the control of neuronal activity in vivo.

By using a combination of cuttingedge genetic engineering, electrophysiological and optical methods, it was possible to remotely control neuronal activity. This technology might be the first stepping stone leading to non-invasive methods for the treatment of neurological disorders.

Claudia Wiedemann

ORIGINAL RESEARCH PAPER Zhang, F. et al. Multimodal fast optical interrogation of neural circuitry. Nature 446, 633-639 (2007) 